\title{
A woman with a rare p.Glu74Gly transthyretin mutation presenting exclusively with a rapidly progressive neuropathy: a case report
}

Anne Schänzer ${ }^{1 *}$, Christoph Kimmich², Christoph Röcken ${ }^{3}$, Thomas Haverkamp ${ }^{4}$, Isabell Weidner ${ }^{4}$, Till Acker $^{1}$ and Heidrun $\mathrm{H} \mathrm{Krämer}^{5}$

\begin{abstract}
Introduction: Familial amyloid polyneuropathy is a rare autosomal dominant disorder caused by mutations in the transthyretin gene, TTR. Diagnosis can be challenging, especially if other family members are not affected or an obvious systemic involvement is lacking. The patients are often misdiagnosed, leading to a delay in the initiation of therapy.

Case presentation: A 35-year-old woman of Turkish origin presented to our outpatient clinic with severe polyneuropathy associated with distally pronounced tetraparesis and hypesthesia of 2 to 3 years' duration. In addition, small nerve fiber involvement with impaired detection of cold temperatures and tingling pain in the lower legs was reported. She did not complain of autonomic dysfunction or visual disturbance. Her family history was empty regarding neuromuscular disorders. The routine diagnostic work-up was unremarkable. A sural nerve biopsy disclosed amyloid deposits, which led to the identification of a rare heterozygous transthyretin mutation (p.Glu74Gly; old classification: p.Glu54Gly).

Conclusions: Few cases with this very heterozygous mutation can be found in the literature. In contrast to the case of our patient, all of the previously described patients in the literature presented with additional severe autonomic symptoms, involvement of the eyes and a positive family history. In this case report, we emphasize that, in patients with progressive neuropathy with small fiber involvement, an amyloid neuropathy should be considered in the differential diagnosis, even if the family history is empty and other organs are not affected.
\end{abstract}

\section{Introduction}

Amyloid polyneuropathies are characterized by pathological deposits of misfolded proteins and peptides in a $\beta$-pleated sheet conformation, which induce damage and a subsequent loss of large- as well as small-diameter nerve fibers. Familial amyloidoses are rare diseases, especially in nonendemic areas, and are divided into three subtypes that present with different involvement of the peripheral nervous system: transthyretin (TTR), apolipoprotein A-I and gelsolin. TTR-associated familial amyloid polyneuropathy (TTR-FAP) is the most common type, in which amyloid depositions in peripheral nerves lead to a progressive sensorimotor and autonomic neuropathy. TTR-FAPs are

\footnotetext{
* Correspondence: anne.schaenzer@patho.med.uni-giessen.de 'Institute of Neuropathology, Justus Liebig University, Arndstrasse 16, 35392 Giessen, Germany

Full list of author information is available at the end of the article
}

autosomal dominant disorders with a variable age of onset between 30 and 70 years, as well as an early ( $<50$ years) and late ( $>50$ years) onset of presentation. A rapidly progressive polyneuropathy is described in most cases. More than 100 mutations in the TTR gene are known, with Val50Met (p.Val30Met) being the most common one. The clinical manifestation can be very heterogeneous; thus, the diagnosis is difficult and might be delayed, especially if the patient is young and the family history negative [1-7]. Early diagnosis is important because treatment options are available. In this report, we describe the clinical, electrophysiological, histopathological and genetic findings in a Turkish woman with a rapidly progressive type of TTRFAP. To the best of our knowledge, this report is the first in the literature describing a patient who presented exclusively with severe neuropathy as a symptom of the rare p.Glu74Gly mutation. 


\section{Case presentation}

A 35-year-old woman of Turkish origin presented to our outpatient clinic with increasing gait and stance disturbances of 2 or 3 years' duration. She reported tingling pain in the feet and lower legs as well as an impairment in detecting cold temperatures as a sign of small fiber involvement. In addition, the patient had recurrent shortness of breath. She had previously been diagnosed with a severe polyneuropathy of unknown origin. Besides the neuropathy, her medical history was empty. She denied gastrointestinal or visual disturbances.

She was a daughter of nonconsanguineous parents from Turkey. Her family history was empty regarding neuromuscular disorders. She has four brothers and two sisters. She is the mother of two healthy children (ages 5 and 7 years).

Her clinical examination revealed a distal as well as left pronounced tetraparesis, hyporeflexia with absence of Achilles tendon reflexes, hypesthesia of the lower legs reaching the patellae and pallanesthesia at the medial malleoli. No compound motor unit potential could be measured on her lower extremities of the tibial or peroneal nerves bilaterally. Motor conduction studies of her upper extremities, including investigation of both the median and ulnar nerves, were unremarkable. No sensory nerve action potentials could be obtained on the upper or lower extremities. Signs of denervation in the distal muscles of the lower extremities, as well as signs of chronic neurogenic reorganization, were detected by electromyography. The extensive polyneuropathy work-up and rheumatology assessments did not produce any pathological results. No indication for a paraneoplastic origin of the disease was discovered. Standard screening methods did not reveal an associated monoclonal gammopathy of undetermined significance or diabetes. However, proteinuria was discovered in the 24-hour urine collection, indicating renal involvement. Because she had dyspnea, a cardiac work-up was performed. In this clinical examination, minor signs of chronic heart failure were found, and we classified her as New York Heart Association functional class II. Magnetic resonance imaging of her heart revealed early left ventricular hypertrophy with a normal ejection fraction of $60 \%$. Echocardiography showed an increased thickness of the interventricular septum of $15 \mathrm{~mm}$ and a slightly impaired longitudinal left ventricular function. The general weakness might also have been associated with an involvement of the autonomic nervous system with low systolic blood pressure (generally $90 \mathrm{mmHg}$ or lower). The patient did not complain about visual disturbances. However, upon examination, opacity of the vitreous body with signs of visual loss was detected in both eyes.

A sural nerve biopsy was performed and processed using standard protocols [8]. Paraffin-embedded nerve tissue was processed for Congo red staining. The stain depicted small but distinct endoneural deposits of amyloid (Figure 1A) with a bright green birefringence under polarizing filters (data not shown). These deposits stained positive with antibodies against transthyretin (TTR) (Figure 1B). Elastica van Gieson staining allowed visualization of collagen fibers and showed a strong endoneural fibrosis (data not shown). Immunohistochemistry against neurofilament and $p$-phenylenediamine-stained semithin sections revealed peripheral nerve fascicles with severe loss of myelinated fibers (about 95\% compared to healthy controls) and only few remaining myelinated fibers (Figures $1 \mathrm{C}$ and 1D). Single fibers with acute myelin degradation could be observed. Electron microscopy confirmed the severe axonal neuropathy with few remaining myelinated fibers with signs of axonal atrophy, few myelin debris and denervated Schwann cell bands (Figures 1E and 1F). Additionally, a severe loss of unmyelinated fibers could be detected. Single amyloid deposits were demonstrated by ultrastructural analysis. There were no signs of axonal regeneration.

In the genetic analyses of the TTR gene, we found a heterozygous missense mutation: c.221A $>$ G (GAG>GGG; p.Glu74Gly). The four coding exons of TTR, corresponding to coding cDNA nucleotides c. 1 to c.444, including flanking sequences, were amplified by PCR and screened for mutations by direct sequencing (reference sequence: TTR ENSG00000118271 with transcript ENST00000237014). Mutation designation according to standard naming conventions of the Human Genome Variation Society [9], which uses numbering beginning at the Met initiation codon, includes a 20-amino acid signal sequence and may be different from that reported in the literature. The alternative mutation designation for the mutation detected in our patient is p.Glu54Gly.

Treatment with tafamidis was initiated after TTR-FAP was diagnosed [10], but our patient's neuropathy was aggravated nonetheless. An orthotopic liver transplant was performed 10 months later, but the patient died as a result of intra-operative complications. Three of her four brothers and three sisters have since presented to our outpatient clinic. A 36-year-old brother tested positive for amyloid in a subcutaneous fat aspiration test. Further family members in Turkey have been evaluated for the mutation. One cousin was found to be a mutation carrier; unfortunately, we have no further information on his health condition.

\section{Discussion}

Hereditary amyloidoses with a predominant involvement of the peripheral nervous system are rare and often difficult to diagnose, especially when other family members are not affected. The patients are often misdiagnosed, leading to a delay in the initiation of therapy. In our patient, a rapidly progressive neuropathy with initial symptoms of small 

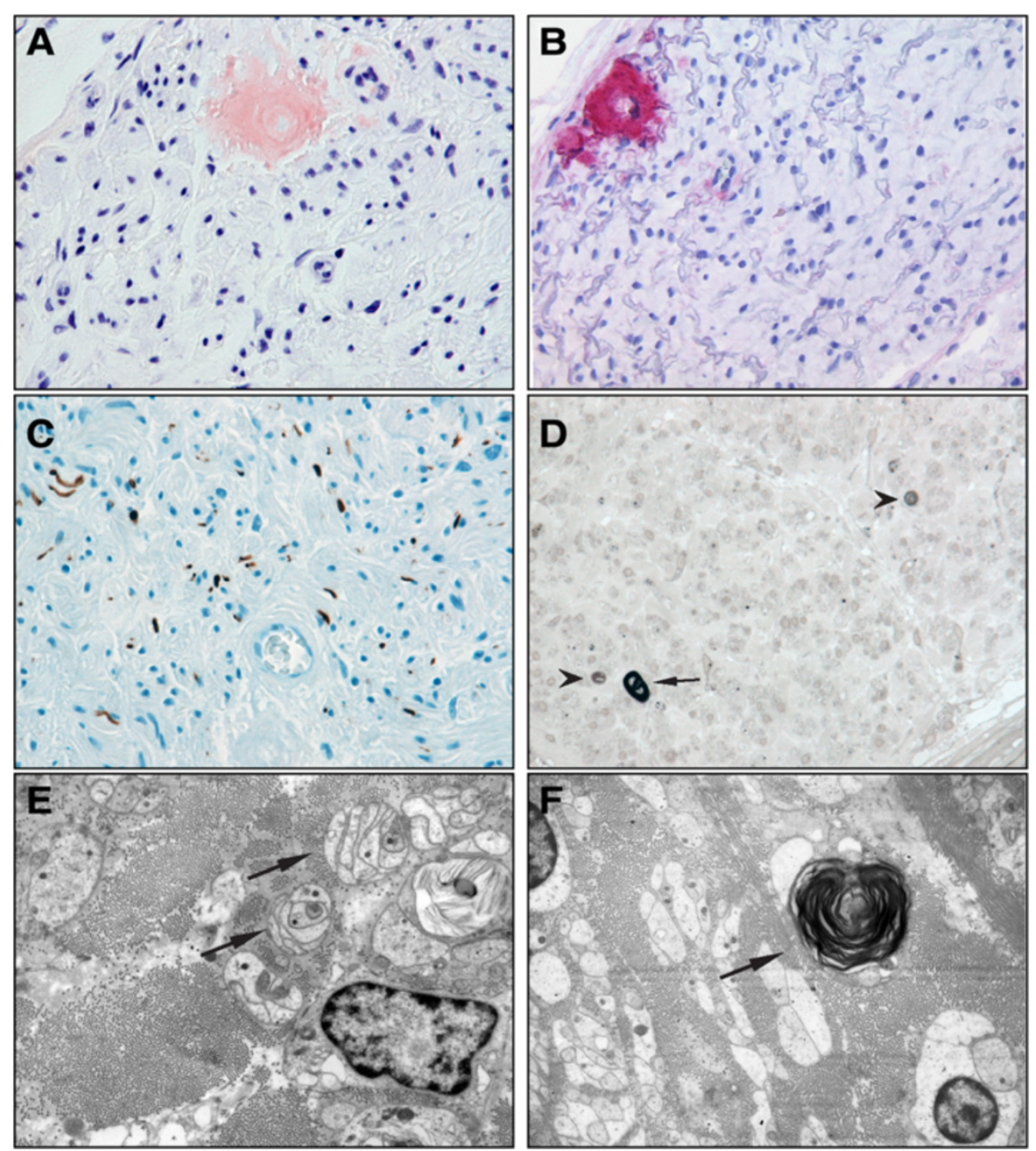

Figure 1 Sural nerve biopsy sections. Small endoneural amyloid deposits are shown in the Congo red staining (A) with a positive transthyretin antibody reaction (B). There is a severe axonal loss with antibodies against neurofilament (C). In the p-phenylenediamine-stained semithin sections, only a few remaining myelinated axons (arrow) can be seen, and acute myelin degeneration (arrowhead) is present (D). Electron microscopic images show denervated Schwann cell bands (arrows) (E) and axonal degeneration with myelin debris (arrow) (F).

fiber involvement was the leading symptom. The major findings were the nerve biopsy with endoneural amyloid deposits and a severe loss of myelinated and unmyelinated fibers in the absence of signs of regeneration.

Some rare mutations of familial amyloid neuropathies are associated with an early onset and a rapid disease progression. Patients with the rare mutation in p.Glu74Gly (pGlu54Gly) usually present with a positive family history and have severe autonomic disturbance causing gastrointestinal symptoms, such as vomiting, constipation and diarrhea, of many years' duration [6,11,12]. Moreover, blindness is an early phenomenon in patients with a mutation in Glu54Gly [11,12]. Our patient did not have any of these symptoms. Although she did not complain about visual disturbances, opacity of the vitreous body with signs of visual loss could be detected.
In this report, we present the case of a 35-year-old woman of Turkish origin who had TTR-FAP with a rare mutation with a phenotype different from those in other cases reported in the literature so far. First, our patient's family history was unremarkable. Second, she reported small fiber involvement in the sense of a small fiber neuropathy without typical autonomic signs. Early involvement of small nerve fibers (A $\delta$ and $C$ fibers) in TTR-FAP patients is well described in the literature [3,7,13-15]. However, the typical autonomic disturbances described in patients with a mutation in Glu54Gly, such as gastrointestinal symptoms, were completely absent in our patient. Third, no visual disturbances were present; however, upon examination, opacity of the vitreous body could be detected. Therefore, the severe polyneuropathy was the only symptom of this very rare mutation in our patient. 


\section{Conclusion}

An amyloid neuropathy should be considered in patients with progressive neuropathies, even if the neuropathy is the only complaint and the patient's family history is negative.

\section{Consent}

Written informed consent was obtained for publication of this case report from the three tested brothers of our deceased patient. Information regarding the other sibling was obtained through the three brothers and the patient. A copy of the written consent is available for review by the Editor-in-Chief of this journal.

\section{Abbreviations}

TTR-FAP: Transthyretin-associated familial amyloidotic polyneuropathy.

\section{Competing interests}

The authors declare that they have no competing interests.

\section{Authors' contributions}

AS and TA performed the histological examination of the nerve. AS was a major contributor to the writing of the manuscript. HK analyzed and interpreted the patient data regarding the neurological disorder and was a major contributor to the writing of the manuscript. CR performed the immunohistological examination of the sural nerve. TH and IW performed genetic analyses. CK analyzed the patient data. All authors read and approved the final manuscript.

\section{Acknowledgments}

We thank Gudrund Schmidt, Nga Rötering and Cornelia Dampmann for their technical assistance.

\begin{abstract}
Author details
${ }^{1}$ Institute of Neuropathology, Justus Liebig University, Arndstrasse 16, 35392 Giessen, Germany. ${ }^{2}$ Medical Department V, Amyloidosis Center, University of Heidelberg, Im Neuenheimer Feld 410, 69120 Heidelberg, Germany. ${ }^{3}$ Institute of Pathology, Christian-Albrechts-University, Arnold-Heller-Strasse 3, 24105 Kiel, Germany. ${ }^{4}$ Laboratory Medicine Dortmund, MVZ Dr Eberhard \& Partner Dortmund, Brauhausstrasse 4, 44137 Dortmund, Germany. ${ }^{5}$ Department of Neurology, Justus Liebig University, Klinikstrasse 33, 35385 Giessen, Germany.
\end{abstract}

Received: 23 May 2014 Accepted: 6 October 2014

Published: 4 December 2014

\section{References}

1. Adams D, Lozeron P, Lacroix C: Amyloid neuropathies. Curr Opin Neurol 2012, 25:564-572.

2. Benson MD, Kincaid JC: The molecular biology and clinical features of amyloid neuropathy. Muscle Nerve 2007, 36:411-423.

3. Planté-Bordeneuve VL, Said G: Familial amyloid polyneuropathy. Lancet Neurol 2011, 10:1086-1097.

4. Russo M, Mazzeo A, Stancanelli C, Di Leo R, Gentile L, Di Bella G, Minutoli F, Baldari S, Vita G: Transthyretin-related familial amyloidotic polyneuropathy: description of a cohort of patients with Leu64 mutation and late onset. J Peripher Nerv Syst 2012, 17:385-390.

5. Schönland SO, Hegenbart U, Bochtler T, Mangatter A, Hansberg M, Ho AD, Lohse P, Röcken C: Immunohistochemistry in the classification of systemic forms of amyloidosis: a systematic investigation of 117 patients. Blood 2012, 119:488-493.

6. Reilly MM, Adams D, Booth DR, Davis MB, Said G, Laubriat-Biachin M, Pepys MB, Thomas PK, Harding AE: Transthyretin gene analysis in European patients with suspected familial amyloid polyneuropathy. Brain 2005, 118:849-856.

7. Dohrn FM, Röcken C, De B, Martin JJ, Vorgerd M, Van den Bergh PY, Ferbert A, Hinderhofer K, Schröder JM, Weis J, Schulz JB, Claeys KG: Diagnostic hallmarks and pitfalls in late-onset progressive transthyretin-related amyloid-neuropathy. J Neurol 2013, 260:3093-3108.

8. Sommer CL, Brandner S, Dyck PJ, Harati Y, LaCroix C, Lammens M, Magy L, Mellgren SI, Morbin M, Navarro C, Powell HC, Schenone AE, Tan E, Urtizbera A, Weis J: Peripheral Nerve Society Guideline on processing and evaluation of nerve biopsies. J Peripher Nerv Syst 2010, 15:164-175.

9. den Dunnen JT, Antonararakis SE: Mutation nomenclature extensions and suggestions to describe complex mutations: a discussion. Hum Mutat $2000,15: 7-12$

10. Coelho T, Maia LF, Martins da Silva A, Waddington Cruz M, Planté-Bordeneuve V, Lozeron P, Suhr OB, Campistol JM, Conceição IM, Schmidt HH, Trigo P, Kelly JW, Labaudinière R, Chan J, Packman J, Wilson A, Grogan DR: Tafamidis for transthyretin familial amyloid polyneuropathy: a randomized, controlled trial. Neurology 2012, 79:785-792.

11. Kim HS, Kim SM, Kang SW, Jung SC, Lee KS, Kim TS, Choi YC: An aggressive form of familial amyloid polyneuropathy caused by a Glu54Gly mutation in the transthyretin gene. Eur J Neurol 2005, 12:657-659.

12. O'Hearn TM, Fawzi A, He S, Rao NA, Lim JL: Early onset vitreous amyloidosis in familial amyloidotic polyneuropathy with a transthyretin Glu54Gly mutation is associated with elevated vitreous VEGF. Br J Ophthalmol 2007, 91:1607-1609.

13. Kim DH, Zeldenrust SR, Low PA, Dyck PJ: Quantitative sensation and autonomic test abnormalities in transthyretin amyloidosis polyneuropathy. Muscle Nerve 2009, 40:363-370.

14. Conceição I, Costa J, Castro J, de Carvalho M: Neurophysiological techniques to detect early small-fiber dysfunction in transthyretin amyloid polyneuropathy. Muscle Nerve 2014, 49:181-186.

15. Lefaucheur JP, Ng Wing Tin S, Kerschen P, Damy T, Planté-Bordeneuve V: Neurophysiological markers of small fibre neuropathy in TTR-FAP mutation carriers. J Neurol 2013, 260:1497-1503.

doi:10.1186/1752-1947-8-403

Cite this article as: Schänzer et al:: A woman with a rare p.Glu74Gly transthyretin mutation presenting exclusively with a rapidly progressive neuropathy: a case report. Journal of Medical Case Reports 2014 8:403.

\section{Submit your next manuscript to BioMed Central and take full advantage of:}

- Convenient online submission

- Thorough peer review

- No space constraints or color figure charges

- Immediate publication on acceptance

- Inclusion in PubMed, CAS, Scopus and Google Scholar

- Research which is freely available for redistribution 